$8-2020$

Prevalence of risk factors of acute kidney injury in a tertiary care hospital in Pakistan

Ainan Arshad

Ahmed Ayaz

Follow this and additional works at: https://ecommons.aku.edu/pakistan_fhs_mc_med_intern_med

Part of the Gastroenterology Commons, and the Internal Medicine Commons 


\section{Prevalence of risk factors of acute kidney injury in a tertiary care hospital in Pakistan}

Ainan Arshad ${ }^{1}$, Ahmed Ayaz ${ }^{2}$

\begin{abstract}
The objective of this study was to determine the prevalence of preventable risk factors of acute kidney injury in a tertiary care hospital in Pakistan. All patients admitted at Aga Khan University Hospital, Karachi with diagnosis of acute kidney injury were included out of which 134 were selected via random sampling. Patients with existing CKD were excluded. Data was then collected retrospectively from medical records of these patients. Mean age was $60 \pm 11.7$ years while mean serum creatinine on admission was $2.4 \pm 1.3(\mathrm{mg} / \mathrm{dl})$. Sepsis played a role in almost half of the patients [60 (45\%)]. Other factors included diarrhoea [23 (17\%)], nephrotoxic drug use [25 (19\%)] and cardiac pathology [24 (18\%)]. It was found that most cases of AKI were due to preventable factors (infections, diarrhoea, and drug toxicity) and concerted efforts to eliminate them would be vital in reducing mortality caused by AKI in developing countries. Keywords: Acute kidney injury, acute renal failure, sepsis https://doi.org/10.5455/JPMA.20286
\end{abstract}

\section{Introduction}

Acute kidney injury is a serious health concern affecting 13.3 million people globally and causing more than 2 million deaths annually. ${ }^{1}$ It has wreaked havoc in patients of all ages around the world as it is independently associated with a poor quality of life and increased risk of mortality. Recent studies have reported alarming figures with incidence as high as $22 \%$ of all hospital admissions and mortality rates above $50 \%$ in severely affected patients. ${ }^{2}$ The International Society of Nephrology (ISN) has addressed this predicament by launching the 0 by 25 initiative which aims to eliminate preventable deaths caused by acute kidney injury in developing countries by the year 2025.2 It intends to achieve this massive feat by introducing policies to timely identify and treat potentially reversible causes of AKI in these countries. Sadly, this campaign can only reach its full potential when comprehensive data from these regions is available 1 Department of Medicine, Aga khan University, Karachi, Pakistan; 25th Year MBBS Student, Medical College, Aga Khan University, Karachi, Pakistan. Correspondence: Ainan Arshad. e-mail: ainan_arshad@hotmail.com regarding $\mathrm{AKI}$ and its risk factors. Some studies have been done in these regions but to our knowledge, none of them used the international KDIGO criteria as per ISN's indications. This study could serve as a platform for larger scale nation-wide studies in Pakistan to tackle this serious issue and help avert millions of needless deaths in Pakistan on a country level.

\section{Patients, methods and results}

This was a single center, retrospective study conducted at Aga Khan University Hospital, Karachi. The study was approved by the Ethics and Review Committee of Aga Khan University. All adult patients admitted at Aga Khan University Hospital, Karachi with diagnosis of acute kidney injury during the period January-December 2017 were included, out of which 134 were selected via random sampling. Patients with existing CKD were excluded. Data was then collected retrospectively from medical records of these patients with recorded variables including demographic data (age and sex), medical history, comorbid conditions, use of nephrotoxic drugs, creatinine at baseline and on admission, urine output, diarrhoea, sepsis, circulatory shock, burns, trauma, diabetes mellitus, hypertension, coronary artery disease, congestive heart failure, neoplasms and HIV infections. The baseline creatinine considered was the last stable measurement in the patient's clinical history prior to admission or, considering that some individuals had no recent or previous creatinine values, the lowest creatinine value within the next 28 days after AKI.

AKI and CKD were diagnosed and classified according to the KDIGO criteria. 3,4 Nephrotoxic drugs [non-steroidal anti-inflammatory drugs (NSAIDs), Antibiotics, angiotensinreceptor blockers (ARBs), angiotensin-converting enzyme inhibitors (ACEIs) and diuretics] were considered the cause of renal failure if a patient had received any of them for a minimum of 3 days prior to the defined increase in serum creatinine concentration. Sepsis was defined by SCCM (Sepsis 1) by the presence of any two or more of the following: Positive blood culture, fever more than $100^{\circ} \mathrm{F}$ $\left(37.5^{\circ} \mathrm{C}\right)$, White cell count $>10 \times 10^{9} / \mathrm{L}$, Heart rate $>90$ beats per minute, Respiratory rate $>20$ breaths $/ \mathrm{min}$,

Vol. 70, No. 8 , August 2020 
Hypotension MAP $<70 \mathrm{mmHg}$, Altered mental status with GCS $<12.5$

During this period 8140 patients were admitted in the department of medicine, out of which 2758 patients were diagnosed with $\mathrm{AKI}, 1418$ were excluded due to CKD. Out of the remaining patients, 134 patients were then selected via random sampling for detailed review of risk factors. (figure). There were 74 males and 60 females with a mean age of $60 \pm 11.7$ years. Baseline characteristics, distribution of KDIGO staging and prevalence of risk factors are shown in Table. Incidence of AKI was $34 \%$ of all hospital admissions. Baseline renal function was known in 127 (95\%) patients with mean creatinine value of $0.90 \mathrm{mg} / \mathrm{dl}$ \pm 0.18 . In 7 (5\%) patients baseline renal function was not available. Sepsis played a role in almost half of the patients [60 (45\%)]. Culture negative sepsis was found in 25 (42\%)

Table: Baseline characteristics of patients and prevalence of risk factors of AKI.

\begin{tabular}{|c|c|}
\hline CHARACTERISTICS & n (\%) \\
\hline $\begin{array}{l}\text { Age range (years) } \\
18-30 \\
31-40 \\
41-50 \\
51-60 \\
61-70 \\
71-80 \\
>80\end{array}$ & $\begin{array}{r}60 \pm 11.7 \\
4(3 \%) \\
9(7 \%) \\
7(5 \%) \\
34(25 \%) \\
68(50 \%) \\
10(8 \%) \\
2(2 \%)\end{array}$ \\
\hline $\begin{array}{l}\text { Gender } \\
\text { Male } \\
\text { Female }\end{array}$ & $\begin{array}{l}74(55.2 \%) \\
60(44.8 \%)\end{array}$ \\
\hline Diabetes & $65(48.5 \%)$ \\
\hline Hypertension & $91(67.9 \%)$ \\
\hline Smokers & $18(13.4 \%)$ \\
\hline Ischaemic heart disease & $48(35.8 \%)$ \\
\hline $\begin{array}{l}\text { Baseline renal function } \\
\text { Mean serum creatinine (mg/dl) }\end{array}$ & $0.90 \pm 0.18$ (Normal range: $0.6-1.2 \mathrm{mg} / \mathrm{dl}$ ) \\
\hline $\begin{array}{l}\text { Admission renal function } \\
\text { Mean serum creatinine (mg/dl) }\end{array}$ & $2.4 \pm 1.3$ \\
\hline $\begin{array}{l}\text { KDIGO STAGE } \\
\text { Stage } 1 \\
\text { Stage } 2 \\
\text { Stage } 3\end{array}$ & $\begin{array}{l}44 \% \\
31 \% \\
25 \%\end{array}$ \\
\hline RISK FACTORS & n (\%) \\
\hline Sepsis & $60(45 \%)$ \\
\hline Gram positive organisms & $6(10 \%)$ \\
\hline Gram negative organisms & $23(38 \%)$ \\
\hline Culture negative & $25(42 \%)$ \\
\hline Culture not done & $6(10 \%)$ \\
\hline Diarrhoea & $23(17 \%)$ \\
\hline Cardiovascular event & $24(18 \%)$ \\
\hline Nephrotoxicity & $25(19 \%)$ \\
\hline NSAIDS & $6(24 \%)$ \\
\hline ACEI/ARBS & $7(28 \%)$ \\
\hline Antibiotics & $4(16 \%)$ \\
\hline Diuretics & $8(32 \%)$ \\
\hline Traumatic injury/RTA & $6(4.5 \%)$ \\
\hline Burns & $1(0.7 \%)$ \\
\hline
\end{tabular}

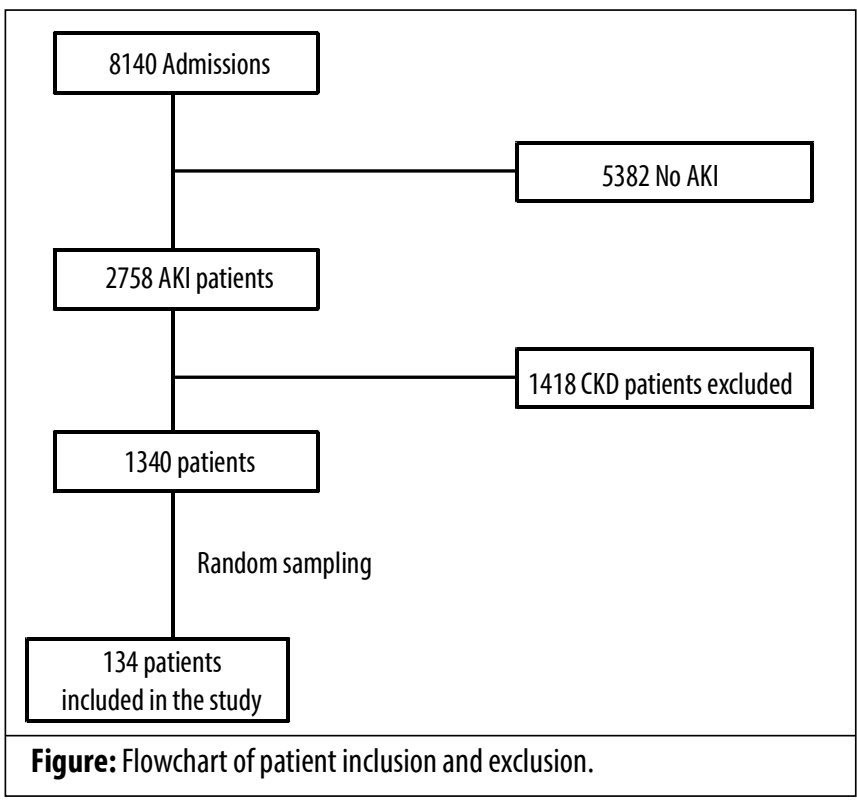

patients and 23 (38\%) patients were infected by gram negative organisms out of which E. Coli was the leading organism. Gram positives were responsible for $10 \%$ of the infections. Other factors leading to AKI included diarrhoea in [23 (17\%)], nephrotoxic drug use in $25(19 \%)]$ and cardiac pathology in24 (18\%)]. The most common age group for all these above pathologies was 61-70 years. Hydronephrosis was not a contributing factor in any of the cases.

\section{Discussion}

AKI in sepsis is significantly associated with a poor prognosis and a higher risk of ICU and hospital mortality.6,7 The growing population of patients that develop AKI due to sepsis, along with the associated ominous prognosis highlight the need for timely management of infections and continuous monitoring of renal function in sepsis in developing countries. Timely diagnosis, prompt haemodialysis, and sufficient supportive therapy have proved to be highly effective in minimizing infectious disease-associated AKI and improving patient outcomes. ${ }^{8}$ Thus, the early diagnosis of AKI and prompt management of infections are a key step forward in the struggle to minimize deaths by this disease.

Diarrhoea was another risk factor of AKI with a prevalence of $23 \%$. This is easily the most preventable cause of AKI anywhere in the world. Prevention starts at the primary care level with timely assessment of patients at risk and adequate rehydration to prevent hypovolaemia. If not 
addressed on time, the consequences tend to be severe. Bradshaw and colleagues, in their national level study in the US, reported that AKI complicates diarrhoeal illness in 1 in 10 patients and is significantly associated with a 5 times increase in mortality. ${ }^{9}$ In addition to this, diarrhoea is already rampant in these parts of the world with biological contamination of drinking water being a major contributor. Appropriate hygiene and cost-effective water filter techniques can play a major role in reducing the spread of diarrhoea and its complications.

Judicious use of nephrotoxic drugs is another worrying yet easily avoidable risk factor of AKI. Our study reported that $19 \%$ of all AKI patients were found to be using nephrotoxic drugs prior to admission. The most common agents in our study were NSAIDS (24\%), ACEI/ARBS (28\%), antibiotics (16\%) and diuretics (32\%). Many elderly patients present with multiple comorbid conditions for which they are usually receiving treatment regimens. They often consult multiple physicians of different specialties for their illnesses, which often leads to infrequent or minimal data sharing between healthcare providers and different clinical settings. Without a unified medical record database, this can lead to overdosing of medications. Another problem in developing countries is the relatively relaxed approach towards prescriptive medications as they are easily obtainable at any common drugstore. Furthermore, pharmacists are rarely recruited at most drug stores, leading to unqualified people selling medications with little knowledge of indications, side effects or drug interactions of different drugs. The best method to minimize this major risk factor is to get thoroughly acquainted with medications that routinely cause nephrotoxicity and to only prescribe such medications after careful evaluation of the patient's comorbid conditions and risk status. Of the 24 (18\%) patients that had cardiovascular events, acute MI was the cause in 14 patients and congestive heart failure in 10 patients.

Deaths associated with AKI is a global health concern, but most preventable deaths occur in low income countries which consist of $85 \%$ of the universal population. ${ }^{10}$

\section{Conclusion}

The results of this study have proved that the majority of risk factors of $\mathrm{AKI}$ are preventable (infections, diarrhoea and drug toxicity) and can be easily eliminated by interventions at the primary level if managed on time. These efforts would go a long way in reducing mortality caused by AKI. Hence, we hope this study becomes the basis for further large-scale studies in Pakistan where AKI continues to wreak havoc and take millions of needless lives.

Ethical Review: The study was approved by the Ethical Review Board of Aga Khan University.

Disclaimer: None.

Conflict of interest: None.

Funding source: None.

\section{References}

1. Li Q, Zhao M, Du J, Wang X. Outcomes of renal function in elderly patients with acute kidney injury. Clin Interv Aging. 2017; 12:15360.

2. Mehta R, Diego S, Burdmann EA. International Society of Nephrology's 0by25 initiative for acute kidney injury (zero preventable deaths by 2025): A human rights case for nephrology. Lancet. 2015; 385:2616-43.

3. Khwaja A. KDIGO Clinical Practice Guidelines for Acute Kidney Injury. Nephron Clin Pract. 2012; 120:c179-84.

4. Erratum: Kidney Disease: Improving Global Outcomes (KDIGO) CKDMBD Update Work Group. KDIGO 2017 Clinical Practice Guideline Update for the Diagnosis, Evaluation, Prevention, and Treatment of Chronic Kidney Disease-Mineral and Bone Disorder (CKD-MBD). Kidney Int Suppl. 2017; 7:1-59.

5. Gül F, Arslanta? MK, Cinel ?, Kumar A. Changing Definitions of Sepsis. Turk J Anaesthesiol Reanim. 2017; 45:129-38.

6. Bagshaw SM, George C, Bellomo R, Database A. Early acute kidney injury and sepsis?: a multicentre evaluation. Crit Care. 2008; 12:R47.

7. Matejovic M, Chvojka J, Radej J, Ledvinova L, Karvunidis T, Krouzecky A, et al. Sepsis and acute kidney injury are bidirectional. Contrib Nephrol. 2011;174:78-88.

8. Cerdá J, Bagga A, Kher V, Chakravarthi RM. The contrasting characteristics of acute kidney injury in developed and developing countries. Nat Clin Pract Nephrol. 2008; 4:138-53.

9. Bradshaw C, Zheng Y, Silver SA, Chertow GM, Long J, Anand S. Acute Kidney Injury Due to Diarrheal IIIness Requiring Hospitalization?: Data from the National Inpatient Sample. J Gen Intern Med. 2018; 33:1520-7.

10. Lewington AJ, Cerdá J, Mehta RL. Raising awareness of acute kidney injury: a global perspective of a silent killer. Kidney Int. 2013; 84:45767. 